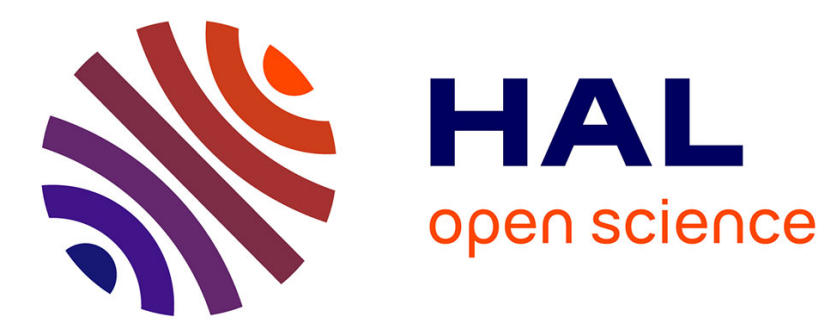

\title{
A Runtime Framework for Energy Efficient HPC Systems Without a Priori Knowledge of Applications
}

Ghislain Landry Tsafack Chetsa, Laurent Lefèvre, Jean-Marc Pierson, Patricia Stolf, Georges da Costa

\section{- To cite this version:}

Ghislain Landry Tsafack Chetsa, Laurent Lefèvre, Jean-Marc Pierson, Patricia Stolf, Georges da Costa. A Runtime Framework for Energy Efficient HPC Systems Without a Priori Knowledge of Applications. 18th International Conference on Parallel and Distributed Systems (ICPAD 2012), Nov 2012, Singapour, Singapore. pp.660-667, 10.1109/ICPADS.2012.94 • hal-00793685

\section{HAL Id: hal-00793685 \\ https://hal.inria.fr/hal-00793685}

Submitted on 22 Feb 2013

HAL is a multi-disciplinary open access archive for the deposit and dissemination of scientific research documents, whether they are published or not. The documents may come from teaching and research institutions in France or abroad, or from public or private research centers.
L'archive ouverte pluridisciplinaire HAL, est destinée au dépôt et à la diffusion de documents scientifiques de niveau recherche, publiés ou non, émanant des établissements d'enseignement et de recherche français ou étrangers, des laboratoires publics ou privés. 


\section{A Runtime Framework for Energy Efficient HPC Systems Without a Priori Knowledge of Applications}

\author{
Ghislain Landry Tsafack Chetsa, Laurent Lefevre \\ INRIA, LIP Laboratory (UMR CNRS, ENS, INRIA, UCB) \\ ENS Lyon, Université de Lyon \\ Lyon, France \\ Email: \{ghislain.landry.tsafack.chetsa, laurent.lefevre\}@ens-lyon.fr
}

\author{
Jean-Marc Pierson, Patricia Stolf, Georges Da Costa \\ IRIT (UMR CNRS) \\ Université de Toulouse \\ Toulouse, France \\ Email: \{pierson, stolf,dacosta\}@irit.fr
}

\begin{abstract}
The rising computing demands of scientific endeavours often require the creation and management of High Performance Computing (HPC) systems for running experiments and processing vast amounts of data. These HPC systems generally operate at peak performance, consuming a large quantity of electricity, even though their workload varies over time. Understanding the behavioural patterns (i.e., phases) of HPC systems during their use is key to adjust performance to resource demand and hence improve the energy efficiency.

In this paper, we describe (i) a method to detect phases of an HPC system based on its workload, and (ii) a partial phase recognition technique that works cooperatively with on-the-fly dynamic management. We implement a prototype that guides the use of energy saving capabilities to demonstrate the benefits of our approach. Experimental results reveal the effectiveness of the phase detection method under real-life workload and benchmarks. A comparison with baseline unmanaged execution shows that the partial phase recognition technique saves up to $15 \%$ of energy with less than $1 \%$ performance degradation.
\end{abstract}

Keywords-energy efficiency; green leverage; phase recognition; execution vector

\section{INTRODUCTION}

The increasing reliance of scientific endeavours on computing has made High Performance Computing (HPC) mainstream in many scientific areas, including climate research, disease control, and drug discovery. Although HPC systems deliver tremendous peak performance for solving challenges faced by the humanity, their electrical consumption and high operational cost have become major concerns. As a consequence, different from a decade ago when supercomputers were ranked only by their peak performance ${ }^{1}$, nowadays they are also assessed based on their energy efficiency ${ }^{2}$.

Improving the energy efficiency of HPC is not trivial as it cannot use classical energy saving capabilities employed in other types of systems because they often reduce the peak performance; which is usually unacceptable in HPC. For example, reducing the CPU frequency or switching off nodes may reduce energy consumption, but depending on the workload this reduction comes at the expense of decreasing

\footnotetext{
${ }^{1}$ http://www.top500.org

${ }^{2}$ http://www.green500.org
}

performance. It is indispensable to choose the right energy saving capability to the workload being executed.

During its life-cycle an HPC application commonly exhibits several behaviours, hereafter referred to as phases or regions, during which techniques can be applied to reduce the electrical consumption of the computing infrastructure. For example, by knowing that the system is running a compute intensive task, a resource manager can spin disks down or put the network interfaces in low power consumption mode. Discovering the task dependency graph can enable optimisations such as reducing processor frequency when running a task that is not in the critical path. This a priori knowledge about applications is often regarded important to improve the energy efficiency of HPC systems. In reality, however, HPC systems are generally shared by multiple applications with heterogeneous computational behaviours, where an optimisation for saving energy considering a single application is likely to impact the performance of others. In addition, the increasing complexity of HPC applications makes it challenging to optimise the energy efficiency per application (a detailed discussion is provided in Section II).

In this work, we overcome this complexity by improving the energy efficiency considering the HPC infrastructure itself (which can be shared by multiple applications) rather than focus on optimising individual applications. We propose and implement an online methodology for phase detection and identification in HPC systems. We then introduce a partial phase recognition technique that guides the usage of green capabilities/leverages. We define a green capability as any action that can save energy in an HPC system, such as: CPU frequency scaling, spinning down disks, scaling the speed of network interconnections, and task migration. An important feature of our methodology is that it does not require prior knowledge of the applications running on the system.

The paper is organised as follows: Section II presents related work. Section III describes our online framework, whereas Section IV presents and analyzes experimental results, and validates the system prototype. Finally, Section $\mathrm{V}$ concludes the paper and discusses future work. 


\section{RELATED WORK}

Over the past few years, several energy saving capabilities have been proposed; they can work either at the hardware level or at the software level. Considering hardware, most vendors provide equipments with techniques to modify the performance of processors, network interfaces, memory, and I/O during their operation, thus allowing for reducing the energy consumed by HPC subsystems. For instance, modern processors are provided with Dynamic Resource Sleeping (DRS), which makes components hibernate to save energy and wakes them up on demand. Although progress has been made, advances in hardware solutions to reduce energy consumption have been slow due to the high costs of equipment design and the increasing demand for performance. Our work combines a set of hardware technologies to reduce the energy consumption of HPC systems.

Unlike hardware approaches, software solutions for reducing the energy usage of HPC systems have gained momentum with the research community. Rountree et al. [1] use node imbalance to reduce the overall energy consumption of a parallel application. They track successive MPI communication calls to divide the application into tasks composed of a communication portion and a computation portion. A slack occurs when a processor waits for data to arrive during a task execution. The speed of the processor can be slowed down with almost no impact on the overall execution time of the application. Rountree et al. developed Adagio to track task execution slacks and compute the appropriate frequency at which a processor should run. Although the first instance of a task is always run at the highest frequency, further instances of the task are executed at the frequency computed after the first execution. In addition, a tool called Jitter [2] was developed to detect slack moments in performance to perform inter-node imbalance and use DVFS to adjust the CPU frequency. Different from Adagio, our fine-grained data collection can differentiate not only computation-intensive and communication-intensive execution portions (which we call phases/regions) but also memory-intensive portions. Memory-intensive phases can be run on a slower core without paying a significant performance penalty [3].

Online techniques have been used to detect and characterise application execution phases, and set the appropriate CPU frequency [4], [5]. The techniques rely on hardware monitoring counters to compute runtime statistics such as cache hit/miss ratio, memory access counts, and retired instructions counts; which are then used for phase detection and characterisation. The developed policies tend to be designed for single task environment [4], [5]. We overcome this limitation by treating each node of a cluster as a black box, which means that we do not focus on any application, but instead on the platform. The flexibility provided by this assumption enables us to track not the applications/workloads execution phases, but nodes' execution phases. Our work also differs from previous work by using partial phase recognition instead of phase prediction. Online recognition of communication phases in an MPI application was investigated by Lim et al. [6]. Once a communication phase is recognised, the authors apply CPU DVFS to save energy. They intercept and record the sequence of MPI calls during program execution and consider a segment of program code to be reducible if there are highly concentrated MPI calls or if an MPI call is long. The CPU is then set to run at the appropriate frequency when the reducible region is recognised again.

To the best of our knowledge, our work differs from those above in two major ways. First, our phase detection approach does not rely on a specific HPC subsystem or MPI communication calls. Second, our model does not focus on saving only the processor energy; it also takes advantage of other power saving capabilities available on HPC subsystems.

The closest research to this work is probably our previous paper [7] in which we developed a cross platform methodology for detecting and characterising phases in HPC systems. Although both efforts use performance counters, they differ significantly; in our previous work we modelled the entire system's runtime as a state graph associated with a transition matrix. The transition matrix helps determine the next state in the graph. The problem with our previous approach is that it takes time to get a useful transition matrix since the system may enter different configurations (which we call letters) from one execution to another, which may prevent it from working well under a system with varying workloads. Moreover, the entire model is built offline.

\section{Methodology}

\section{A. Global Approach}

The rationale behind our methodology is that it is possible to save energy while maintaining performance by selecting the most suitable green capabilities for the system at a phase. Approaches described in Section II set the CPU frequency according to the estimated use of the processor over a time interval. In addition to scaling the CPU frequency, an HPC system can potentially benefit from other energy reduction schemes conceived to adjust the system to the actual demand,. These schemes include adjusting the speed of the network interconnect; switching off memory banks; spinning down disks; migrating tasks across nodes.

A typical HPC system exhibits many different phases or behaviours during runtime, complicating the choice of green capabilities. Characterising phases at runtime, so that similar patterns can be easily identified in the future, is hence worthwhile. With this characterisation, a set of green capabilities that maintain the performance at a reasonable level (in terms of execution time) and improve the energy efficiency at given phase can be employed later during similar phases. Our approach associates each phase with a characterisation 
and a set of green capabilities. A detailed analysis of phase characterisation is provided in Section III-B.

As mentioned earlier, phase characterisation and identification enable reuse of green capabilities suitable to one phase by another phase. However, a phase can be considered similar to a previous phase once it completes, at which time applying green capabilities related to the former might not produce the expected results. The literature uses prediction to identify an upcoming phase, which requires a priori information about the workload being executed; not appropriate for systems shared by multiple applications. Therefore, as we do not have any a priori knowledge of the workload, we introduce a heuristic referred to as partial phase recognition or simply partial recognition.

Instead of trying to recognise a complete phase prior to adjusting the system (which would then be useless since the phase would have finished), we decide to adjust the system when a fraction of a phase has been recognised. This technique clearly gives false positives (i.e. sections incorrectly recognised as part of a phase), but we argue that adjusting the system is beneficial at least for a certain time. When the current phase diverges too much from the recognised phase, then another phase can be identified or a new phase characterised.

Further details on phase identification and partial recognition are given in Section III-C, whereas Section III-D details how green capabilities are selected, explains how they are linked to phases, and discusses how the proposed approach can be enforced in a distributed system with independent nodes.

\section{B. Defining and Characterizing Phases}

Our approach is based on partial recognition of phases during the system activity. A system phase also known as phase, represents the runtime behaviour of the system over a given interval during which specific metrics are stable; meaning that their corresponding values remain below a given threshold within the phase. These metrics can be application or system specific and are computed for each node using data about the computational state of the node.

To illustrate the idea, let us assume we only have one metric, the load of the system. Consider a system whose load, measured every second, is displayed in Figure 1; where the $\mathrm{x}$-axis shows the time in seconds and the $\mathrm{y}$-axis the load. With a threshold set to $5 \%$, we can easily recognise 4 phases (following the vertical lines in the graphic, from time 0 to 2 , from time 3 to 6 , from time 7 to 9 , and from time 10 to 13). Each of these phases is characterised by its duration and the different loads the system went through during that phase. The number of phases obviously varies with the threshold, for with a threshold of let's say 50\% we would have had 2 phases (from time 0 to 9 , and from time 10 to 13).

As a general rule, a single metric (the load of the system in our example) is not adequate for characterising useful

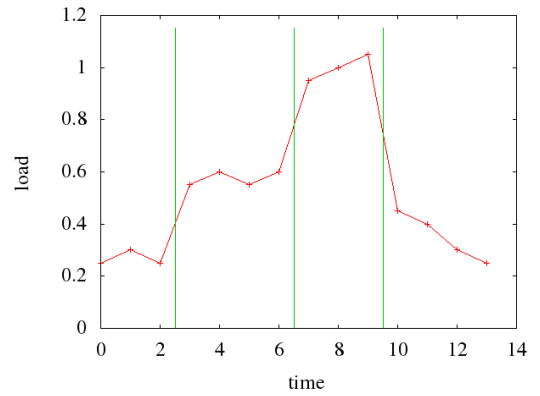

Figure 1. Example of phase characterization based on load

phases. The set of data chosen will be small enough to save space, but adequately large so as to distinguish between different phases.

As stated beforehand, each phase is associated with its duration and data collected during the phase. The size of the data representing a phase is proportional to its duration, which implies that large amounts of data might be collected for very long phases. To prevent this, a digest is used to summarise the data. We define a signature as a representative for a phase, where the signature is computed from multiple values. In the following, the signature will be computed as the vector of averaged monitored values. In the previous example, the signature of a phase can be the average load during the phase.

\section{Phase Identification and Partial Recognition}

1) Execution Vectors: In order to apply any of the green capabilities, it is worth detecting and identifying appropriate phases. An Execution Vector (EV) is a vector of values that represent the behaviour of an application during a time interval. The length of these intervals is defined depending on the goal reactivity. It can be of the order of the mean time to perform an action (switching on or off a node is an action that takes minutes, whereas changing a processor's frequency is at the second time-scale). EVs are considered as points in the positive quadrant of the execution space spanned by the $\mathrm{N}$ vector dimensions; and their values must give a maximum insight on the current execution. These values can be taken from the system (load, network- or I/Orelated) or directly from the hardware using performance counters. Hardware performance counters are special hardware registers originally designed to performance tuning. However, they have recently been used for investigating the power usage of applications [8], [9]. According to the literature, the most important counters are bus cycles, number of instructions, cache misses, cache hits, cache references, and stall cycles (they may have different names depending on the architecture).

2) Phase Detection and Identification: Following the definition of a phase in Section III-B, we can assume without 


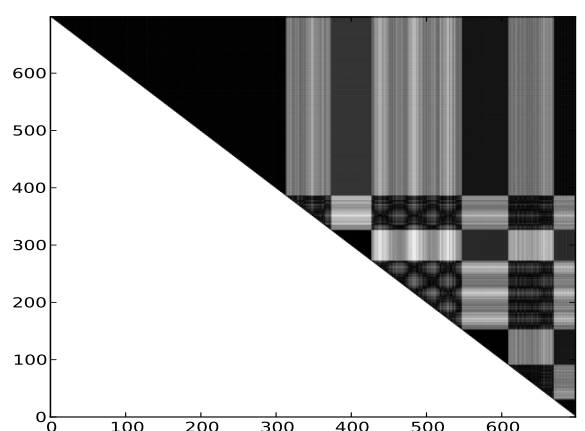

Figure 2. Matrix of distance (manhattan) between execution vectors, where the diagonal represent the execution time line. The darker, the more similar.

loss of generality that during a phase execution vectors are stable.

Figure 2 displays the similarity matrix using execution vectors collected when a cluster was running a synthetic benchmark which successively runs Lower-Upper symmetric Gauss-Seidel (LU), Embarrassingly Parallel (EP), Conjugate Gradient (CG) and Integer Sort (IS) from NPB-3.3 [10] benchmark. A detailed explanation of similarity matrices using power vectors can be found in the literature [11]. As for power vectors, they represent for the whole execution the distance between all pairs of EVs. At coordinate $i, j$ in the upper matrix of Figure 2 the colour represents on a grey scale the distance between the EV at time $i$ and $j$ respectively.

Consequently, the colour at the point $i, j$ tends to black when the distance between $\mathrm{EV}$ at $i$ and $\mathrm{EV}$ at $j$ tends to zero. Similarly, the colour at the position $i, j$ tends to white as the distance between $\mathrm{EV}$ at $i$ and $\mathrm{EV}$ at $j$ increases. Above the diagonal line (the diagonal represents the execution timeline from the upper left corner to the lower right corner), we can report 7 triangular blocs. These blocs represent phases the system went through when executing the workload (our synthetic benchmark). From Figure 2, we can also observe that distances between execution vectors within the same phase approach zero, whereas in between phases the distances approach one.

The above observations led us to the premise that a phase change occurs when the distance between two consecutive EVs goes beyond a given threshold, which we refer to as the detection threshold.

While phase detection can serve any kind of system optimisation, keeping all execution vectors is not realistic. Hence, once a phase completes, it is associated a signature vector. The signature vector of a phase is a vector obtained by computing the arithmetic average of each metric (e.g., load, network, or a particular performance counter) of the vectors computed during the phase. It is used for phase identification, where a phase is considered similar to another if the distance between their signature vectors is below the detection threshold. If the distance between the signature vectors of the new phase and an already identified phase is below the detection threshold, then we do nothing; otherwise a new phase is added via its signature vector to the signature vectors list. Put simply, only the first occurrence of a phase is stored.

3) Partial Recognition: Partial recognition technique is used to identify phases before their completion. Once a phase is completed, in addition to its signature vector and its characteristics, we store its reference vector and its length. The reference vector of a phase is a randomly chosen execution vector among those pertaining to the set of vectors sampled during the phase. Thus, an ongoing phase (a phase started but not completed yet) $P_{\text {ongoing }}$ is recognised as an existing phase $P_{\text {exist }}$ if the manhattan distance between each execution vector of the already executed part of $P_{\text {ongoing }}$ and the reference vector of $P_{\text {exist }}$ is below a given threshold defining the percentage of dissimilarity between them. We state that the recognition threshold is $\mathrm{X} \%$ if the already executed part of $P_{\text {ongoing }}$ equals X\% of the length of $P_{\text {exist }}$.

\section{Using Phases to Save Energy}

Having identified a phase based on information of a previously characterised phase, data on the latter is used for choosing the most relevant green capabilities. The following explains how this approach can be enforced in a distributed system with multiple nodes.

1) Link Between Phases and State: The stability of metrics within a phase implies that the behaviour of the system in a phase is more or less constant; meaning that there is a predominant behaviour within the phase. That predominant behaviour is considered as the state of the phase. Referring to the literature, a phase can be cpu-intensive, memoryintensive, and IO-intensive (including network accesses).

To find out the predominant behaviour of a phase or its state, we apply Principal Component Analysis (PCA) to the dataset made up by execution vectors belonging to the corresponding phase. PCA is typically issued when several underlying factors shape the data. Therefore, we use variables (each variable represents a metric) contributing less to the first principal axis of PCA to determine the predominant behaviour over the phase (components of the execution vector are access rate of our sensor set).

2) Enforcing Green Leverage: The goal is to optimise the energy consumption of HPC systems under the following constraints: (a) energy optimisation must be done without any specific information about the characteristics of the application prior to its execution; (b) management policies for reducing the overall system energy consumption must not have a significant impact on performance.

Given these constraints, the most suitable capabilities have to be selected for each phase. Knowing the state of a phase and its remaining time before completion, system adaptation 


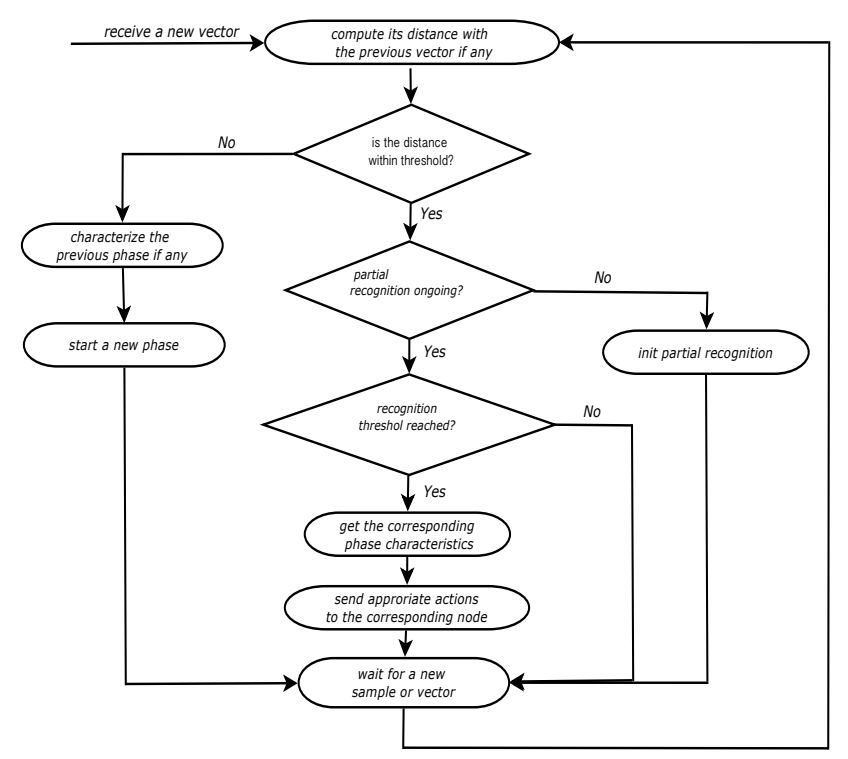

Figure 3. Decision process.

is straightforward via green capabilities. Possible system adaptations are listed bellow.

- Adjusting the system's computational level: (a) high or CPU-bound; (b) medium or memory-bound; (c) low for non-CPU/memory-bound applications and idle systems.

- Adjusting the network: (a) interconnects speed; (b) switching off interconnect equipments.

- Storage/disk availability: (a) switching off memory banks; (b) spinning down disks.

- Task migration: migrating all tasks to a few nodes can cut down the energy usage if the rest of the nodes are switched off.

3) Global Architecture: The diagram of Figure 3 shows the steps of our detection making process. Partial recognition starts as soon as a new phase has begun. When the partial recognition threshold is reached (the ongoing phase is identified with an existing phase), the characteristics (including its duration) of the existing phase are used to adapt the system accordingly. In a distributed system with independent nodes we propose a centralised architecture with a global coordinator. The motivation for the centralised management is that task migration, which is one of our green capabilities, requires a global view of the system. Figure 4 depicts the global architecture. The decision making node implements the decision making process; it sends commands to appropriate nodes after decision making. On each node of the system a process samples one execution vector per second and sends it to the global coordinator.

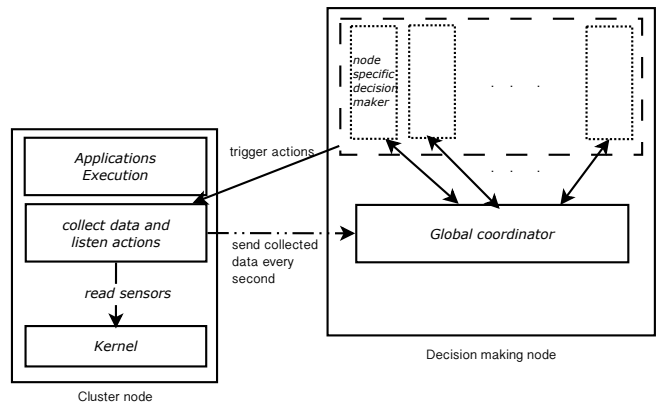

Figure 4. Global coordinator overview.

\section{EXPERIMENTATION AND VALIDATION}

\section{A. Phase Detection and Partial Recognition Results}

1) Phase Detection Results: Phase detection is critical to any management policy that intends to take advantage of workload variability. Analysis of phase detection using power vectors [11] and basic bloc vectors [12] have been provided in the literature. However, these techniques use offline phase analysis. We rely on EVs which are comparable to power vectors [11] used in power phase behaviour identification.

The execution vector represents the access rate of a set of performance counters along with the disk access rate (read/write) and network access rate (send/receive) per second. Each new EV is normalised with respect to a maximum vector. The maximum vector is special in the sense that it comprises the maximum available value of the elements of all execution vectors.

For phase detection, we use the manhattan distance between two consecutive execution points; we then state that two executive EVs belong to the same phase or have the same behaviour if the manhattan distance between them is below a given threshold. The threshold is actually a fixed percentage of the maximum known distance between all consecutive EVs. This maximum is updated any time a greater value is found. Our experiments show that a threshold of 5\% is effective in many cases. Figure 5 where doted vertical lines represent the identified phases shows the results of our online phase detection with a 5\% threshold on one node of our cluster. During the experiment, our cluster was running the Advance Weather Research and Forecasting (WRF-ARW) [13] model. WRF-AWR is globally one phase broken up with very short transition phases. However, as our phase detection process looks at the runtime behaviour (via execution vectors) of each node of the platform and considers as a phase any behaviour between two consecutive behavioural changes, transition phases will cause WRFAWR to break into many micro-phases.

To demonstrate the effectiveness of our online detection approach, we implemented using execution vectors, the offline phase detection approach presented by Isci $e t$ 


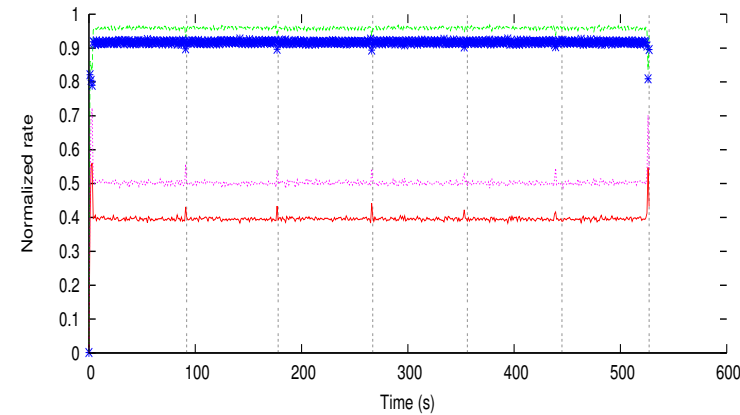

Figure 5. Online phase detection with a 5\% threshold for WRF-ARW.

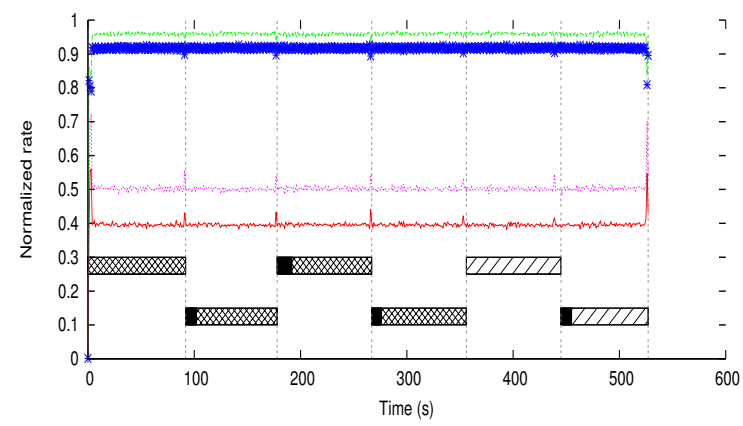

cache-write-op cache-read-op -
netsentbytes instructions

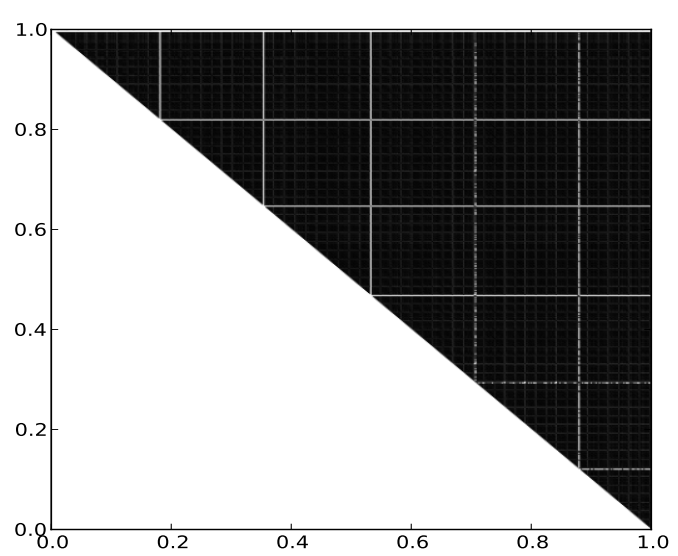

Figure 7. Offline phase detection with a 5\% threshold for WRF-ARW (the matrix corresponds to half of the execution time). The diagonal represents the execution time-line from the upper left corner to the lower right corner.

\section{B. Real-life System Implementation: Measurement and Evaluation Platform}

We implemented our online phase detection and recognition and tested them on a cluster system. The implemented prototype monitors the overall cluster's behaviour via performance monitoring counters along with network bytes sent/received counts and disk read/write counts. For convenience, we use the term "sensors" to designate performance monitoring counters along with network bytes sent/received counts and disk read/write counts. Each node of the cluster sends a sample of data to the decision making node on a per-second basis. Samples collected corresponding to execution vectors are further used by the decision maker node to perform phase detection and partial recognition for any individual node. Partial recognition is more suitable in our case because phases are not detected at fixed intervals; besides, the system may be shared by many applications.

The use of green capabilities available on the managed cluster is guided by our partial phase recognition. When partial recognition is successful, the characteristics of the phase the newly started phase is identified with are translated into a predefined set of "green settings". The system adaptation implemented so far uses CPU frequency scaling as a proof of concept, but we plan to implement many other green leverages/capabilities including task migration, disk sleep state, and network interconnect speed scaling.

1) Dynamic Power Management via Green Leverage/Capabilities: Our phase characterisation relies on the assumption that sensors contributing less to the first principal axis of PCA give more information about what did not happen during the phase. Thus we arbitrarily select 5 sensors among those contributing less to the first principal axis of PCA for phase characterisation. In addition, we compute 
Table I

TRANSLATION OF PHASE TO GREEN SETTINGS (IO RELATED SENSORS INCLUDES NETWORK AND DISK ACTIVITIES).

\begin{tabular}{|c|l|}
\hline $\begin{array}{c}\text { Sensors selected from PCA } \\
\text { for phase characterisation }\end{array}$ & Decisions \\
\hline $\begin{array}{c}\text { bus_cycles \& } \\
\text { cache_misses \& } \\
\text { IO related sensors }\end{array}$ & $\begin{array}{l}\text { CPU frequency sets to its maximum } \\
\text { spin down the disk } \\
\text { network speed scaled down }\end{array}$ \\
\hline no IO related sensor & $\begin{array}{l}\text { CPU frequency sets to its lowest } \\
\text { network speed scaled up }\end{array}$ \\
\hline instructions \& & $\begin{array}{l}\text { CPU frequency set to its minimum } \\
\text { network speed scaled up }\end{array}$ \\
\hline last level cache misses (llc) & $\begin{array}{l}\text { CPU frequency sets to its average value } \\
\text { network speed scaled down } \\
\text { spin down the disk }\end{array}$ \\
\hline IO related sensors & CPU frequency sets to its maximum \\
\hline IO related sensors
\end{tabular}

the average instruction rate of each phase, which is then used for idle phase characterisation. We need such a metric for characterising idle phases as over the course of idle phases almost all sensors are likely to be constant. Table I shows an example of phases translation to green settings. The left column specifies sensors appearing in the set of sensors selected for phase characterisation; whereas, the right column specifies the associated decision. For instance, when sensors selected from PCA for phase characterisation only include $\mathrm{I} / \mathrm{O}$ related sensors, the decision associated is scaling the CPU frequency to its maximum.

2) Evaluation Platform: Our evaluation platform is a fifteen-node cluster set up on the French large-scale experimental platform called Grid5000 ${ }^{3}$. Each node is an Intel Xeon X3440 with 4 cores and 16 GB of RAM. Available frequency steps for each core are: $2.53 \mathrm{GHz}, 2.40 \mathrm{GHz}$, $2.27 \mathrm{GHz}, 2.13 \mathrm{GHz}, 2.00 \mathrm{GHz}, 1.87 \mathrm{GHz}, 1.73 \mathrm{GHz}$, $1.60 \mathrm{GHz}, 1.47 \mathrm{GHz}, 1.33 \mathrm{GHz}$ and $1.20 \mathrm{GHz}$. In our experiments, low computational level always sets the CPU frequency to the lowest available frequency, which is 1.20 $\mathrm{GHz}$, whereas high and medium computational levels set the CPU frequency to the highest available $(2.53 \mathrm{GHz})$ and $2.00 \mathrm{GHz}$ respectively. Each node uses its own hard drive which supports active, ready and standby states. Infiniband$20 \mathrm{G}$ is used for interconnecting nodes. The Linux kernel 2.6.35 is installed on each node where perf event is used to read the hardware monitoring counters. MPICH is used as MPI library. LU, SP, BT from NPB-3.3 [10] and a real life application the Advance Research WFR (WRFARW) model are used for the experiments. Class $C$ of the NAS benchmark is used (compiled with default options). WRF-ARW is a fully compressible conservative-form nonhydrostatic atmospheric model. It uses an explicit timesplitting integration technique to efficiently integrate the Euler equation. We monitored each node power usage on a per second basis using a power distribution unit.

\footnotetext{
${ }^{3}$ http://www.grid5000.fr
}

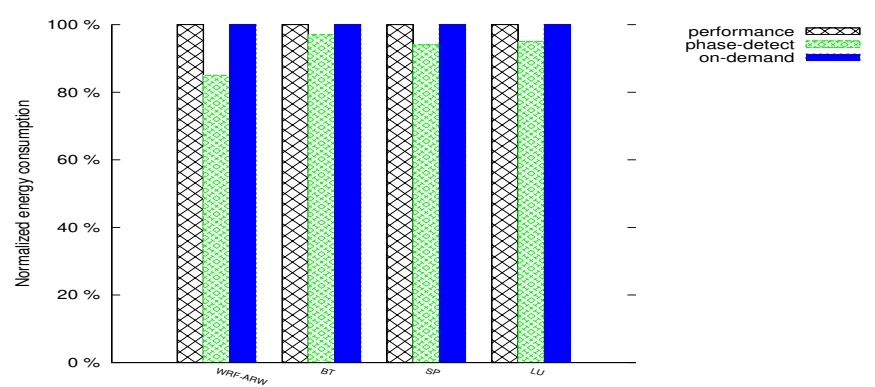

Figure 8. Phase detection and partial recognition guided leverage results: the chart shows the average energy consumption by applications under the three system's configurations.

\section{Experimental Results: Analysis and Discussion}

To evaluate our management policy, we consider 3 basic configurations of the monitored cluster: (a) on-demand configuration in which the Linux "on-demand" governor is enabled on all the nodes of the cluster; (b) performance configuration where each node's CPU frequency scaling governor is set to "performance"; (c) phase-detect configuration in which we detect phases identified using partial recognition and apply green capabilities accordingly.

Histograms of Figure 8 present the normalised average energy consumption of the overall cluster for each application under the three cluster configurations. Figure 9 shows their respective execution time. These figures show that our management policy (phase-detect) consumes in average $15 \%$ less energy than "performance" and "on-demand" while offering the same performance for our real-life application, WRF-ARW. For LU, BT and SP the average energy gain is between $3 \%$ and $6 \%$, as we could not achieve as much energy savings as with WRF-AWR since many phases detected for these applications are compute intensive. From Figure 9, we notice a performance loss of less than $3 \%$ for LU and BT (performance is evaluated in terms of execution time). No need to mention that "on-demand" performs better than "performance" since all the applications we use are CPU intensive. Hence, the CPU load is always above $95 \%$, a situation wherein the on-demand governor remains at the highest frequency.

We do better than a Linux governor because Linux ondemand governor will not scale the CPU frequency down unless the system load decreases below a threshold. The problem at this point is that the CPU load generally remains very high for memory intensive workloads/phases that do not require the full computational power. Thus, the energy gain made with these applications and particularly with WRFAWR results in slowing the CPU frequency down when a node is suspected to be in a memory-bound phase.

Above results demonstrate the effectiveness of our system's energy management scheme based on phase detection and partial recognition. However, there are several underlying issues that need to be addressed in the future. Some of 


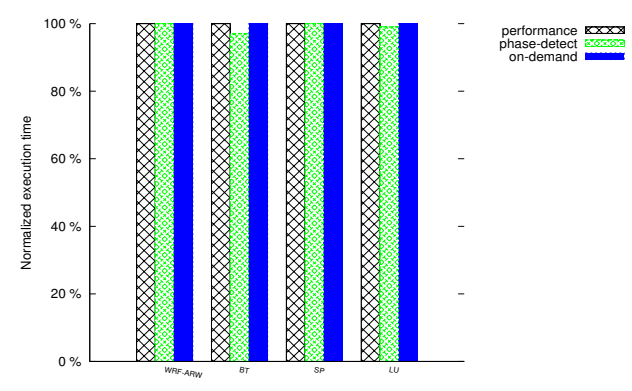

Figure 9. Phase detection and partial recognition guided green leverage results: the chart shows average performance loss incurred by our management policy with respect to "performance" and "on-demand".

these issues are highlighted in this paragraph. Performance counters are not the same on all architectures, which means one may need to find out which sensors are useful on his architecture. Despite this limitation, the system management scheme we introduce here is a general purpose model and can be easily scaled to integrate previous research efforts.

\section{CONCLUSiON}

In this paper, we present an approach to optimise the energy consumed by high performance computing systems at runtime. We propose to detect phases of an HPC system based on its workload. Then, by using a partial phase recognition technique, we predict the green capabilities than should be applied to save energy. This detection and improvements are achieved during runtime without knowing any details about the application. A software prototype has been implemented and validated. Experimental results reveal the effectiveness of our phase detection approach under real-life workload and benchmarks. Comparison with baseline unmanaged execution shows that our partial phase recognition algorithm can guide the use of green capabilities and save up to $15 \%$ of energy with less than $1 \%$ performance degradation. Future work includes integrating workload consolidation along with task migration as core functionalities of our prototype.

\section{ACKNOWLEDGMENT}

This work is supported by the INRIA large scale initiative Hemera focused on "developing large scale parallel and distributed experiments". Some experiments of this article were performed on the Grid5000 platform, an initiative from the French Ministry of Research through the ACI GRID incentive action, INRIA, CNRS and RENATER and other contributing partners (http://www.grid5000.fr). Authors would also like to thank Marcos Dias de Asuncao for his help in improving the readability of this paper.

\section{REFERENCES}

[1] Rountree B, Lowenthal D, Supinski B, Schulz M, Freeh V, Bletsch T, "Adagio: making DVS practical for complex HPC applications," Proceedings of the 23th International Conference on Supercomputing (ICS09), Yorktown Heights, New York, USA. 812 Jun., 2009, pp. 460-469.

[2] Kappiah N, Freeh V, Lowenthal D, "Just in time dynamic voltage scaling: exploiting inter-node slack to save energy in mpi programs," Proceedings of the 19th ACM/IEEE Conference on Supercomputing (SC05), Washington, USA. 12-18 Nov., 2005, pp. 33-41.

[3] R. Kotla, A. Devgan, S. Ghiasi, T. Keller and F. Rawson, "Characterizing the Impact of Different Memory-Intensity Levels," IEEE 7th Annual Workshop on Workload Characterization (WWC-7), Oct. 2004.

[4] C. Isci, G. Contreras, and M. Martonosi, "Live, runtime phase monitoring and prediction on real systems with application to dynamic power management," Proc. MICRO, 2006, pp. 359370 .

[5] K. Choi, R. Soma, and M. Pedram, "Fine-grained dynamic voltage and frequency scaling for precise energy and performance tradeoff based on the ratio of off-chip access to onchip computation times," IEEE Trans. Comput.-Aided Design Integr. Circuits Syst., vol. 24, no. 1, pp. 1828, Jan. 2005.

[6] Lim M, Freeh V, Lowenthal D, "Adaptive, transparent frequency and voltage scaling of communication phases in mpi programs," in proceedings of the 20th ACM/IEEE Conference on Supercomputing (SC06), Tampa, Florida, USA. 11-17 Nov., 2006, pp. 14-27.

[7] G.L. Tsafack, L. Lefevre, J.M. Pierson, P. Stolf, and G.D. Costa, "DNA-inspired Scheme for Building the Energy Profile of HPC Systems," In proceedings of the First International Workshop on Energy-Efficient Data Centres, Madrid, Spain, May 2012.

[8] G. Contreras, "Power prediction for intel xscale processors using performance moni- toring unit events," In proceedings of the International symposium on Low power electronics and design (ISLPED, ACM Press, 2005, pp. 221226.

[9] K. Singh, M. Bhadauria, and S. A. McKee, "Real time power estimation and thread scheduling via performance counters," SIGARCH Comput. Archit. News, 37:4655, July 2009.

[10] D. H. Bailey, E. Barszcz, J. T. Barton, R. L. Carter, T .A. Lasinski, D. S. Browning, L. Dagum, R. A. Fatoohi, P. O. Frederickson, and R. S. Schreiber, "The nas parallel benchmarks. International Journal of High Performance Computing Applications," 1991, 5:6373.

[11] C. Isci and M. Martonosi. Identifying program power phase behavior using power vectors, "In Workshop on Workload Characterization," September 2003.

[12] T. Sherwood, E. Perelman, G. Hamerly and B. Calder, "Automatically Characterizing Large Scale Program Behavior," Proceedings of the Tenth International Conference on Architectural Support for Programming Languages and Operating Systems (ASPLOS X), Oct., 2002, pp 45-57.

[13] W. C. Skamarock, J. B. Klemp, J. Dudhia, D. Gill, D. Barker, W. Wang, J. G. Powers, "A description of the Advanced Research WRF Version 2. NCAR Technical Note," NCAR/TN468+STR (2005). 